\title{
Ropivacaine Hydrochloride
}

National Cancer Institute

\section{Source}

National Cancer Institute. Ropivacaine Hydrochloride. NCI Thesaurus. Code C52198.

The hydrochloride salt of ropivacaine, a local anesthetic of the amide type with analgesic activity. Ropivacaine binds to voltage-gated sodium ion channels in the neuronal membrane, thereby preventing the permeability of sodium ions and resulting in a stabilization of the neuronal membrane and inhibition of depolarization; nerve impulse generation and propagation are blocked, resulting in a reversible loss of sensation. 